\title{
A High-Throughput, Seedling Screen for Plant Immunity
}

\author{
Alexandre Martel, ${ }^{1}$ Timothy Lo, ${ }^{1}$ Darrell Desveaux, ${ }^{1,2, \dagger}$ and David S. Guttman ${ }^{1,2, \dagger}$ \\ ${ }^{1}$ Department of Cell and Systems Biology, University of Toronto, Ontario, Canada \\ ${ }^{2}$ Centre for the Analysis of Genome Evolution \& Function, University of Toronto, Ontario, Canada \\ Accepted 17 December 2019.
}

\begin{abstract}
An understanding of how biological diversity affects plantmicrobe interactions is becoming increasingly important, particularly with respect to components of the pathogen effector arsenal and the plant immune system. Although technological improvements have greatly advanced our ability to examine molecular sequences and interactions, relatively few advances have been made that facilitate high-throughput, in vivo pathology screens. Here, we present a high-throughput, microplate-based, nondestructive seedling pathology assay, and apply it to identify Arabidopsis thaliana effector-triggered immunity (ETI) responses against Pseudomonas syringae type III secreted effectors. The assay was carried out in a 48-well microplate format with spray inoculation, and disease symptoms were quantitatively recorded in a semiautomated manner, thereby greatly reducing both time and costs. The assay requires only slight modifications of common labware and uses no proprietary software. We validated the assay by recapitulating known ETI responses induced by $P$. syringae in Arabidopsis. We also demonstrated that we can quantitatively differentiate responses from a diversity of plant genotypes grown in the same microplate. Finally, we showed that the results obtained from our assay can be used to perform genome-wide association studies to identify host immunity genes, recapitulating results that have been independently obtained with mature plants.
\end{abstract}

Keywords: Arabidopsis thaliana, avirulence factors, effectors, effector-triggered immunity (ETI), Pseudomonas syringae, resistance genes, screen, seedling, type III secreted effector (T3SE), type-3 secretion

A. Martel and T. Lo contributed equally to this work.

D. Desveaux and D. S. Guttman contributed equally to this work.

Accession numbers: zarl-1 (At3g50950; SALK_013297), zed1-2 (At3g57750; EMS line), and zrk2-1 (At3g55720; SAIL_916_G06). Arabidopsis ecotype accession numbers are listed in Supplementary Table S1.

${ }^{\dagger}$ Corresponding authors: D. S. Guttman; david.guttman@utoronto.ca; and D. Desveaux; darrell.desveaux@utoronto.ca

Funding: Support was provided by the Natural Sciences and Engineering Research Council of Canada Discovery Grant and Postgraduate Award, and the Centre for the Analysis of Genome Evolution and Function Canada Research Chairs Comparative Genomics and Plant-Microbe Systems Biology grants.

*The $\boldsymbol{e}$-Xtra logo stands for "electronic extra" and indicates that eight supplementary figures and one supplementary table are published online.

The author(s) declare no conflict of interest.

(c) 2020 The American Phytopathological Society
The study of plant-microbe interactions has been central to deciphering the plant immune system, while providing numerous promising approaches for crop protection. The most intensively studied plant-microbe pathosystem is the interaction between the $\gamma$-proteobacterial pathogen Pseudomonas syringae and the model plant Arabidopsis thaliana (Mansfield et al. 2012). $P$. syringae is a highly diverse species complex that can infect an equally varied spectrum of plant species (Baltrus et al. 2017; Berge et al. 2014; Morris et al. 2013). Research in this area has led to important advances in our understanding of bacterial virulence, plant immunity, and host-pathogen diversity and evolution (Couto and Zipfel 2016; Dillon et al. 2019a,b; Provart et al. 2016; Smakowska-Luzan et al. 2018; Wu et al. 2017; Xin et al. 2018).

$P$. syringae strains, like many bacterial pathogens, carry a diverse and highly variable suite of type III secreted effectors (T3SEs). These T3SEs are injected directly into the host cell through the type III secretion system and have evolved to promote virulence by disrupting the cellular homeostasis or immune response of the host (Büttner 2016; Khan et al. 2018). Plants have responded to pathogenic intracellular effectors by evolving resistance proteins (nucleotide-binding domain leucine-rich repeat receptors [NLRs]) that can recognize the presence or activity of specific effectors. This recognition elicits effector-triggered immunity (ETI), which is a rapid and robust immune response often associated with a localized cell-death response termed the hypersensitive response (HR) (Cui et al. 2015; Jones and Dangl 2006).

HR-based assays have traditionally and commonly been used to identify ETI-eliciting T3SEs-so-called avirulence proteins (Abramovitch et al. 2006; Jones and Dangl 2006; Khan et al. 2018). However, some T3SE elicitors have been found to elicit an ETI response that does not include a visible HR, suggesting that HR-based screens may provide only a partial, and perhaps biased, view of the scope of ETI responses (Jayaraman et al. 2017; Seto et al. 2017). Infection assays that quantify bacterial growth or quantify disease symptoms (Laflamme et al. 2016) provide means to overcome this issue but these methods are often labor intensive in the space and time required for the propagation of mature plants, which ultimately limits their throughput.

Here, we describe a high-throughput, nondestructive, in vivo seedling pathology assay to facilitate the screening of T3SEs that elicit ETI in Arabidopsis. The method uses spray inoculation of seedlings grown in microplates and is readily coupled with automated disease quantification approaches such as Plant Immunity and Disease Image-Based Quantification (PIDIQ) (Laflamme et al. 2016). We demonstrate the utility of the approach by recovering eight published $P$. syringaeArabidopsis ETI responses, regardless of their HR-eliciting capabilities. Furthermore, we illustrate how the method can be used for genome-wide association studies (GWAS) by recapitulating previously published results identifying the Arabidopsis 
RPS5 and RPM1 NLRs as responsible for ETIs against the P. syringae T3SEs HopAR1 and AvrRpm1, respectively (Atwell et al. 2010).

\section{RESULTS}

High-throughput seedling pathology assay workflow.

We developed a high-throughput, nondestructive seedling pathology assay, which we applied to screen $P$. syringae T3SEs for ETI elicitation on Arabidopsis. The screen was carried out in 48-well microplates using plates with perforated wells for easy bottom watering (Supplementary Fig. S1). The modified microplates were filled with sieved soil, which was then compacted by centrifugation (Supplementary Fig. S1). Seed may either be aliquoted into each well manually or through a seed dispenser (modified microplate; Supplementary Fig. S2) to rapidly dispense seed into specific wells across multiple soil-filled plates. Plants were grown in standard Arabidopsis conditions for 1 week, then infected with $P$. syringae using standard spray infection techniques, followed by a second spray infection 2 days following the initial infection (days postinfection [dpi]), which resulted in more pronounced and consistent disease symptom development (Fig. 1) (Seto et al. 2017). Plates were imaged periodically for $10 \mathrm{dpi}$.

To quantify the progression of disease symptoms, we first photographed the microplates and developed an ImageJ macro that crops and labels each individual well (Fig. 1). We used our previously described PIDIQ ImageJ macro to quantify the extent of chlorotic disease symptoms shown by plants in each well, a phenotype contributed to by the $P$. syringae phytotoxin coronatine (Fig. 1) (Laflamme et al. 2016; Mittal and Davis 1995). Because PIDIQ requires the integrity of plant tissue for quantification, only plate images with intact plant tissue for each well were used for PIDIQ, typically 5 to 10 dpi. Later stages of infection with virulent $P$. syringae resulted in macerated plant tissue that was indistinguishable from the soil. Using 48-well microplates greatly increased the amount of data points that could be obtained in the space of our standard plant growth tray $(28$ by $54 \mathrm{~cm}$ ), from 60 (typically 6 rows by 10 columns of mature plants per tray) to 576 (12 plates by 48 wells). Additionally, the time required from seed to analysis was reduced from 6 to 3 weeks using the high-throughput plate assay. Thus, our workflow allowed for quantification of Arabidopsis disease symptoms in a nondestructive manner with substantial savings in labor, time, and space.

\section{Recapitulating characterized ETI elicitors with the high-throughput seedling pathology assay.}

Our primary objective was to rapidly and effectively identify ETI-eliciting $P$. syringae T3SEs. We validated the assay by screening for immunity using previously identified $P$. syringae T3SEs that elicit ETI on Arabidopsis Col-0. We performed spray infections using $P$. syringae pv. tomato DC3000 (PtoDC3000) heterologously expressing the following ETIeliciting T3SEs under the control of their native promoters: HopK1 (AvrRps4) (Gassmann et al. 1999), AvrB (Grant et al. 1995), AvrRpt2 (Kunkel et al. 1993; Yu et al. 1993), HopF2a (Seto et al. 2017), HopAR1 (AvrPphB) (Warren et al. 1998), HopA1 (Kim et al. 2009), AvrRpm1 (Grant et al. 1995), and HopZ1a (Lewis et al. 2010), as well as an empty vector (EV) control. Notably, these T3SEs are catalytically diverse, their ETI elicitation is dependent on several NLRs, and they differ in their HR-eliciting capabilities.

Spray infection with PtoDC3000::EV caused severe chlorosis and death of Arabidopsis Col-0 seedlings, whereas plants infected with PtoDC3000 harboring the T3SEs showed robust
ETI responses characterized by healthy seedlings at both 5 and 10 dpi (Fig. 2A; Supplementary Fig. S3). We used PIDIQ to quantify disease symptoms (i.e., the percentage of tissue that is chlorotic yellow) and confirmed that PtoDC3000 harboring ETI-eliciting T3SEs displayed significantly reduced disease symptoms compared with the PtoDC3000::EV at 5 dpi (Fig. 2B).

\section{Quantifying plant genotype-specific phenotypes with the high-throughput seedling pathology assay.}

To confirm whether the development of disease symptoms could be quantified in a plant genotype-specific manner, we used PtoDC3000 heterologously expressing HopZ1a, which requires the Arabidopsis NLR gene ZARl and the pseudokinase ZED1 to trigger ETI (Lewis et al. 2008, 2010, 2013; Ma et al. 2006). PtoDC3000::HopZ1a was spray inoculated onto a plate containing wild Arabidopsis wild-type Col-0, zarl-1, zed1-2, and $z r k 2-1$, which is a pseudokinase related to and present in the same genomic cluster as ZED1 (Lewis et al. 2013). As expected, chlorotic symptoms developed exclusively on zarl-1 and zed1-2, whereas the remaining genotypes displayed robust ETI responses (Fig. 3A; Supplementary Fig. S4). This observation was confirmed using PIDIQ, where zarl-1 and zed1-2 displayed significantly higher disease symptoms than those present on wild-type Col-0 or zrk2-1 (Fig. 3B). Therefore, our approach can quantify ETI responses in a genotype- and wellspecific manner.

\section{Using the high-throughput seedling pathology assay for GWAS.}

GWAS is a powerful tool that has successfully identified components involved in multiple ETI responses, including the NLR genes RPS5 and RPM1 that recognize the T3SEs HopAR1 and AvrRpm1, respectively (Atwell et al. 2010; Huard-Chauveau et al. 2013); however, screening the number of Arabidopsis ecotypes required for a GWAS can be laborious and time consuming. To determine whether the highthroughput seedling pathology assay is suitable for a GWAS, we screened 110 Arabidopsis ecotypes in duplicate for their ability to mount an ETI response to PtoDC3000::HopAR1 (Fig. 4A and B; Supplementary Fig. S5). We used PIDIQ for symptom quantification and analyzed the data using the online accelerated mixed-model GWAS tool available through the GWA-Portal (Fig. 4B; Supplementary Table S1) (Seren 2018; Seren et al. 2012). The analysis identified a significant peak in chromosome 1, centered on the RPS5 locus, the NLR gene required for HopAR1 ETI (Fig. 4C) (Warren et al. 1998). To further confirm the suitability of our method to be coupled to GWAS, we screened 70 Arabidopsis ecotypes for their ability to mount an ETI response to PtoDC3000::AvrRpm1 (Supplementary Fig. S7). Using the above-described PIDIQ and GWAS methods, a significant peak was identified in chromosome 3, centered on the RPMI locus, the NLR required for AvrRpm1 ETI (Fig. 4D) (Grant et al. 1995). These analyses recapitulate HopAR1 and AvrRpm1 GWAS results that were obtained using HR phenotypes from mature plants (Atwell et al. 2010). Importantly, we demonstrated that successful GWAS may be performed using symptom development rather than HR, expanding the number of ETI elicitors that are amenable to this type of analysis.

\section{DISCUSSION}

We have developed a high-throughput, nondestructive pathology assay for the screening of $P$. syringae T3SEs against Arabidopsis seedlings in a microplate format. Our results demonstrated that published ETI responses to $P$. syringae 
T3SEs are retained in seedlings and recovered by our assay method. The screen dramatically reduces labor, space, time, and costs. For example, it is possible to quantitatively measure host-pathogen interactions within 3 weeks from seed sterilization to final disease symptom quantification. Furthermore, because the method utilizes disease symptom quantification rather than the elicitation of a macroscopic HR, it may capture HR-dependent as well as HR-independent ETI responses.

Alternative methods have been developed to increase the throughput of ETI screens such as the luxCDABE-tagged $P$. syringae strains, which allow for quantification of bacterial growth without performing laborious growth assays (Fan et al.
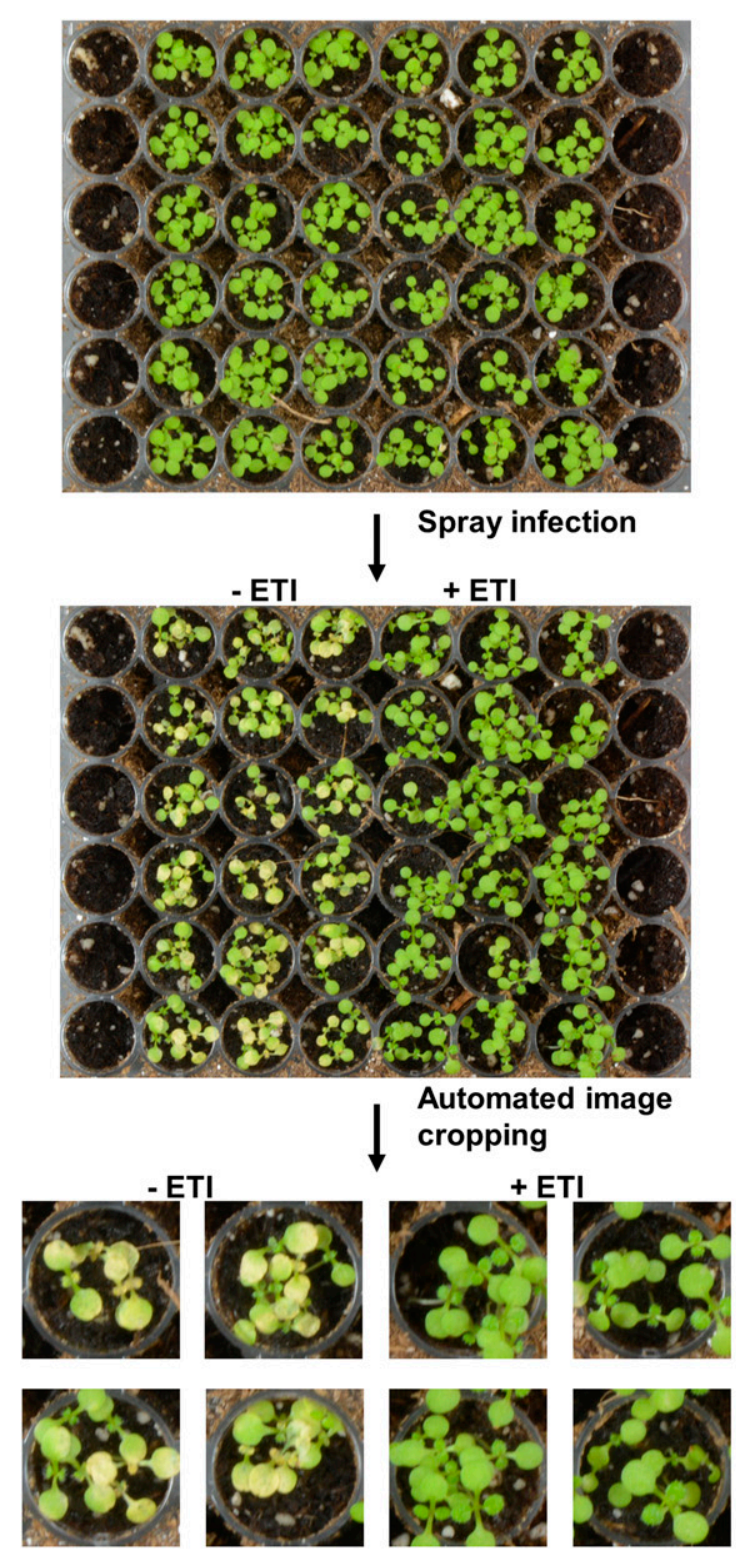

Automated PIDIQ analysis of plant health

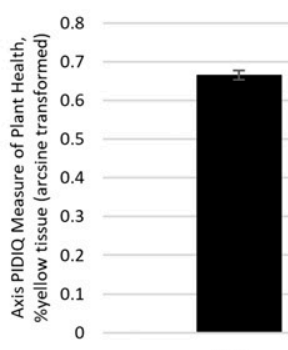

ETI -

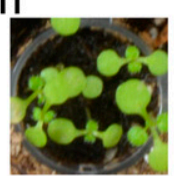

\section{Progression of disease symptoms (5-10 days post infection)}

\section{Cropped images}

\section{Quantitative measure of plant health}

Fig. 1. Overview of the seedling pathology assay. Seeded plates were stratified for 2 days at $4{ }^{\circ} \mathrm{C}$ (not shown), then grown for 7 days before being sprayed with Pseudomonas syringae pv. tomato DC3000. Disease symptoms were monitored daily, with the majority of disease symptoms developing 5 to 10 days postinfection. Plates were photographed and the images were subjected to automatic image cropping before disease symptom quantification using Plant Immunity and Disease Image-Based Quantification (PIDIQ) for downstream analyses. ETI = plus $(+)$ or minus $(-)$ effector-triggered immunity. 
A

Pto DC3000
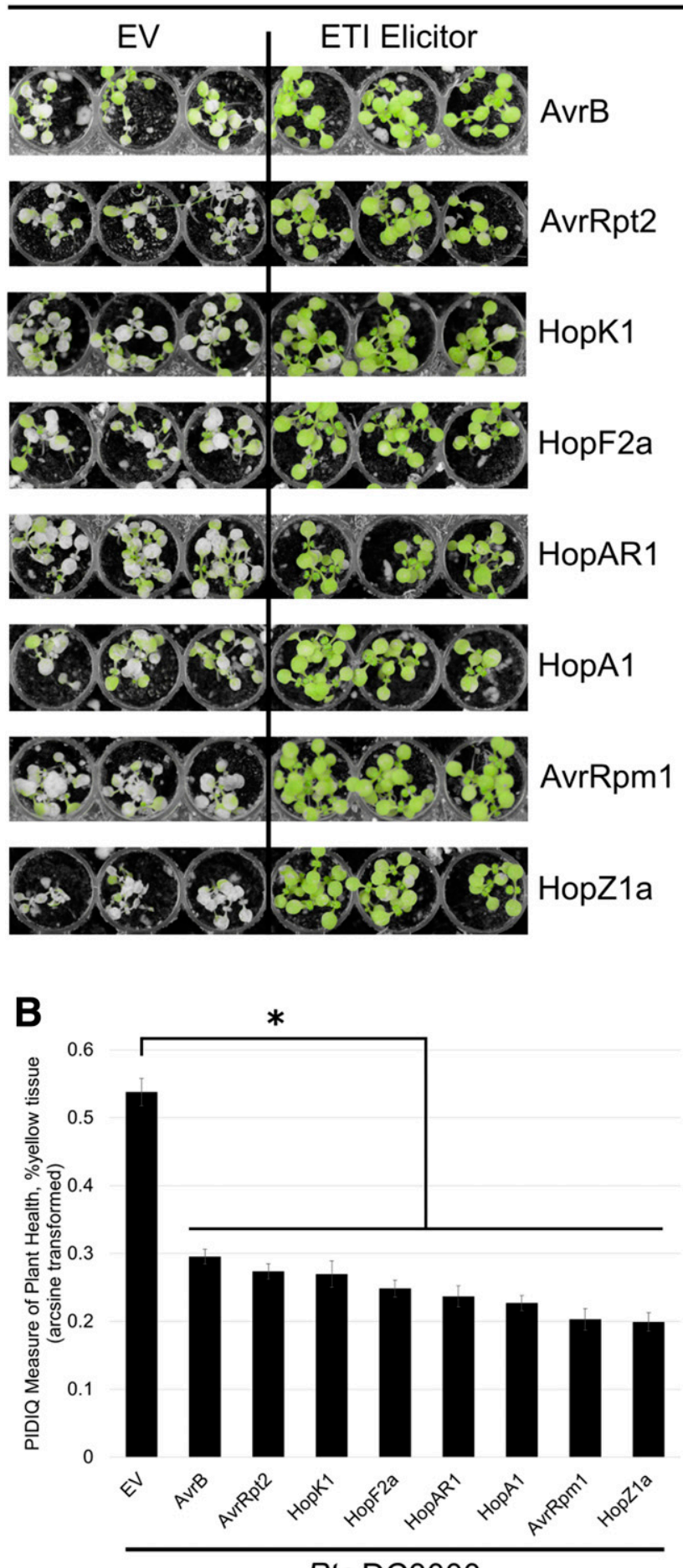

Pto DC3000

Fig. 2. Distinct effector-triggered immunity (ETI) responses are retained in Arabidopsis seedlings. A, Wild-type Arabidopsis Col-0 seedlings were infected with a Pseudomonas syringae pv. tomato DC3000 empty vector (EV) control or 1 of 8 characterized ETI elicitors. Images were taken 5 days postinfection with the Effects green-pass filter that shows healthy green tissue in its natural color and diseased tissue in gray (see Experimental Procedures). Unfiltered plate images are presented in Supplementary Figure S3. B, Disease symptom quantification using Plant Immunity and Disease Image-Based Quantification (PIDIQ) of Arabidopsis Col-0 seedlings 5 days postinfection for each assessed ETI elicitor. Error bars represent standard error $(n=18)$. The asterisk denotes statistically significant differences (Student's $t$ test, $P<0.05$ ).
2008). Although this luminescent system provides an accurate proxy for determining bacterial growth, it remains an end-point assay that requires destruction and manipulation of the plant tissue as well as specialized equipment. In comparison, our microplate-based assay is nondestructive and requires no specialized equipment.

We found that we could readily observe differences between multiple genotypes arrayed in the same microplate, and that these differences could be quantified and recorded in a semiautomated manner to high accuracy. These features greatly facilitate the implementation of GWAS, which requires the screening of a large number of plant genotypes. GWAS approaches have proven successful in identifying genetic components involved in multiple ETI responses with independent mechanisms of recognition (Atwell et al. 2010; Huard-Chauveau et al. 2013). Our HopAR1 and AvrRpm1 GWAS analyses provided results similar to those previously published for these
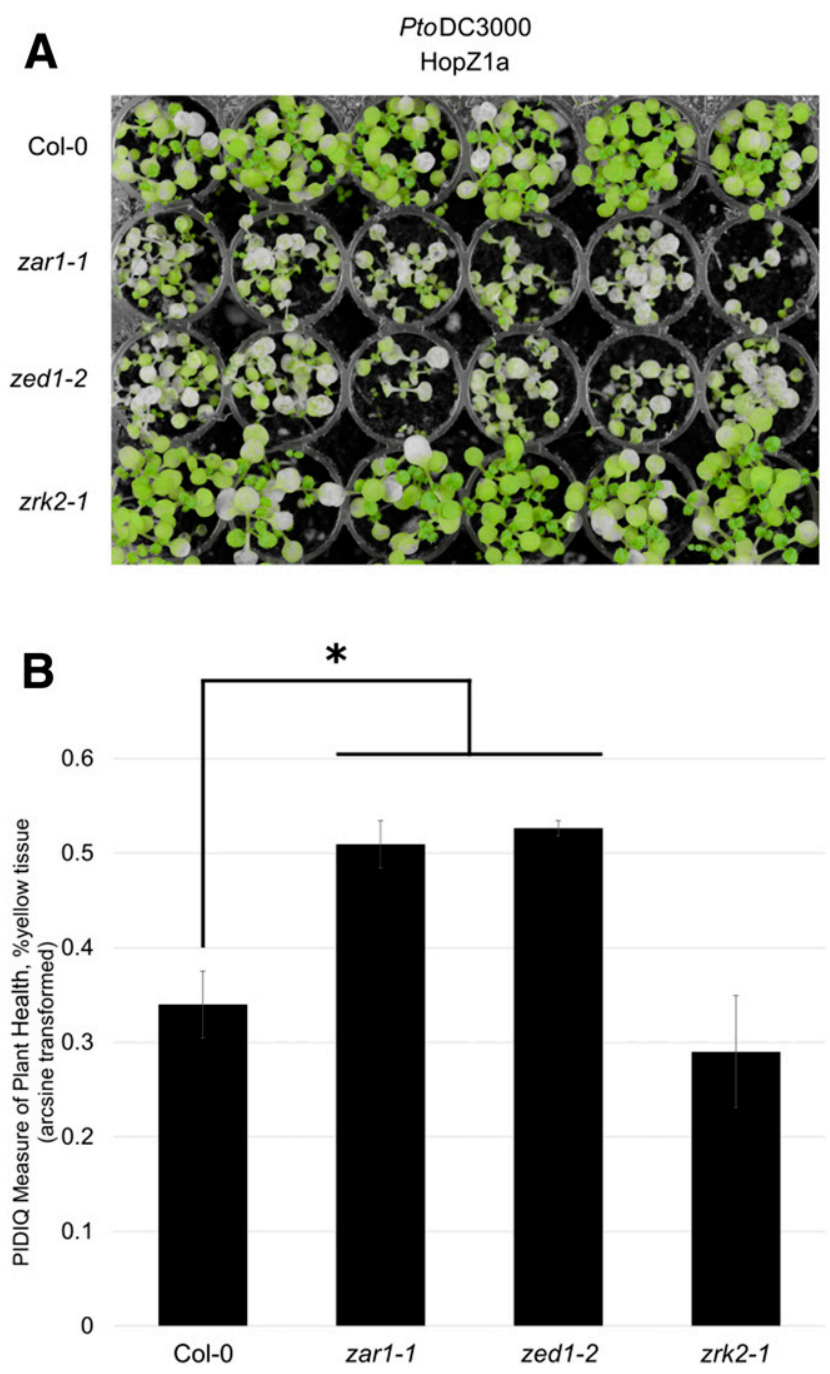

Fig. 3. Genotype-specific disease quantification. A, Arabidopsis Col-0 wild type, zarl-1, zed1-2, and zrk2-1 seedlings were infected with Pseudomonas syringae pv. tomato DC3000 expressing the HopZ1a type III secreted effector. Images were taken 5 days postinfection with the Effects green-pass filter that shows healthy green tissue in its natural color and diseased tissue in gray (see Experimental Procedures). Unfiltered plate images are presented in Supplementary Figure S4. B, Disease symptom quantification using Plant Immunity and Disease Image-Based Quantification (PIDIQ) of infected seedlings 5 days postinfection. Error bars represent standard error $(n=6)$. The asterisk denotes statistically significant differences (Tukey's honestly significant difference, $P<0.05$ ). 
A

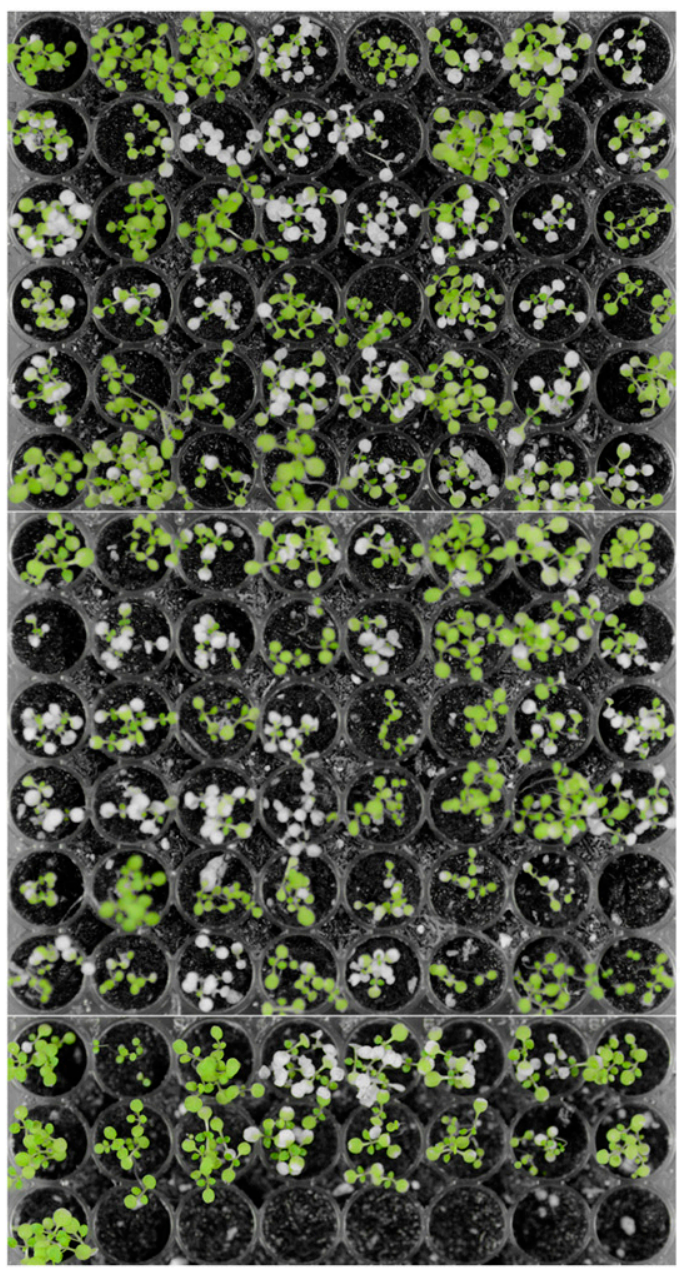

B

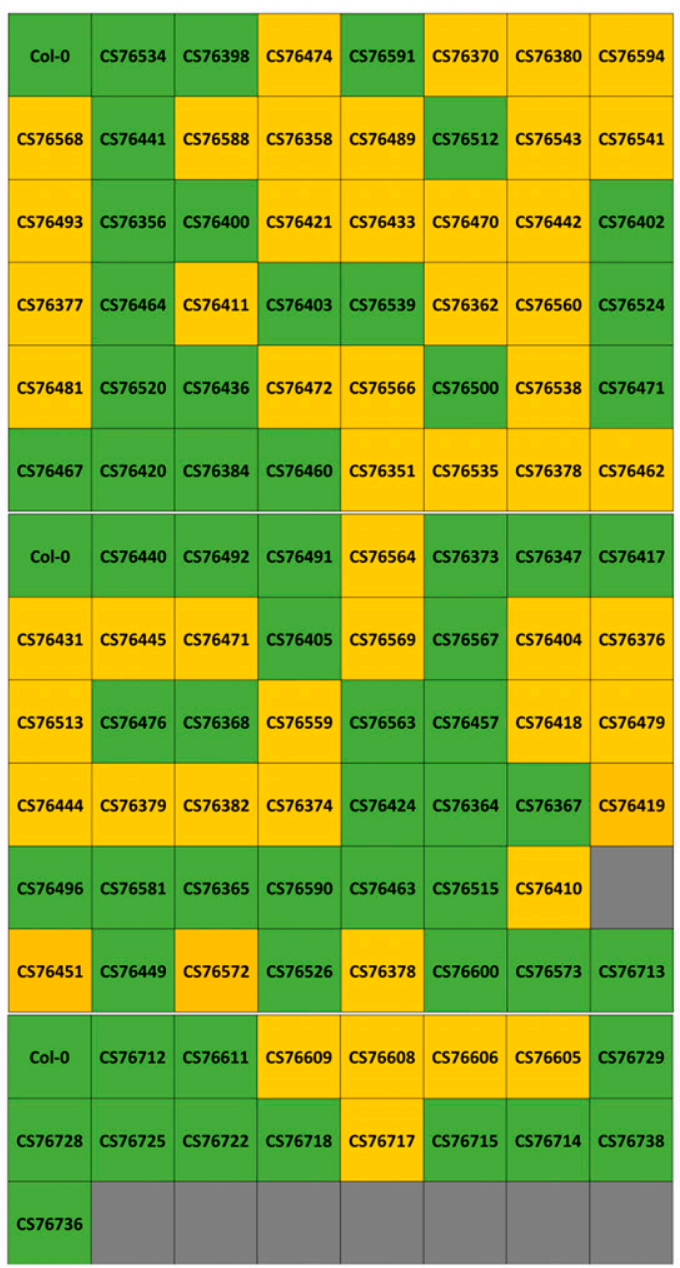

\section{RPSS}

Bonferroni

Benjamini Hochberg
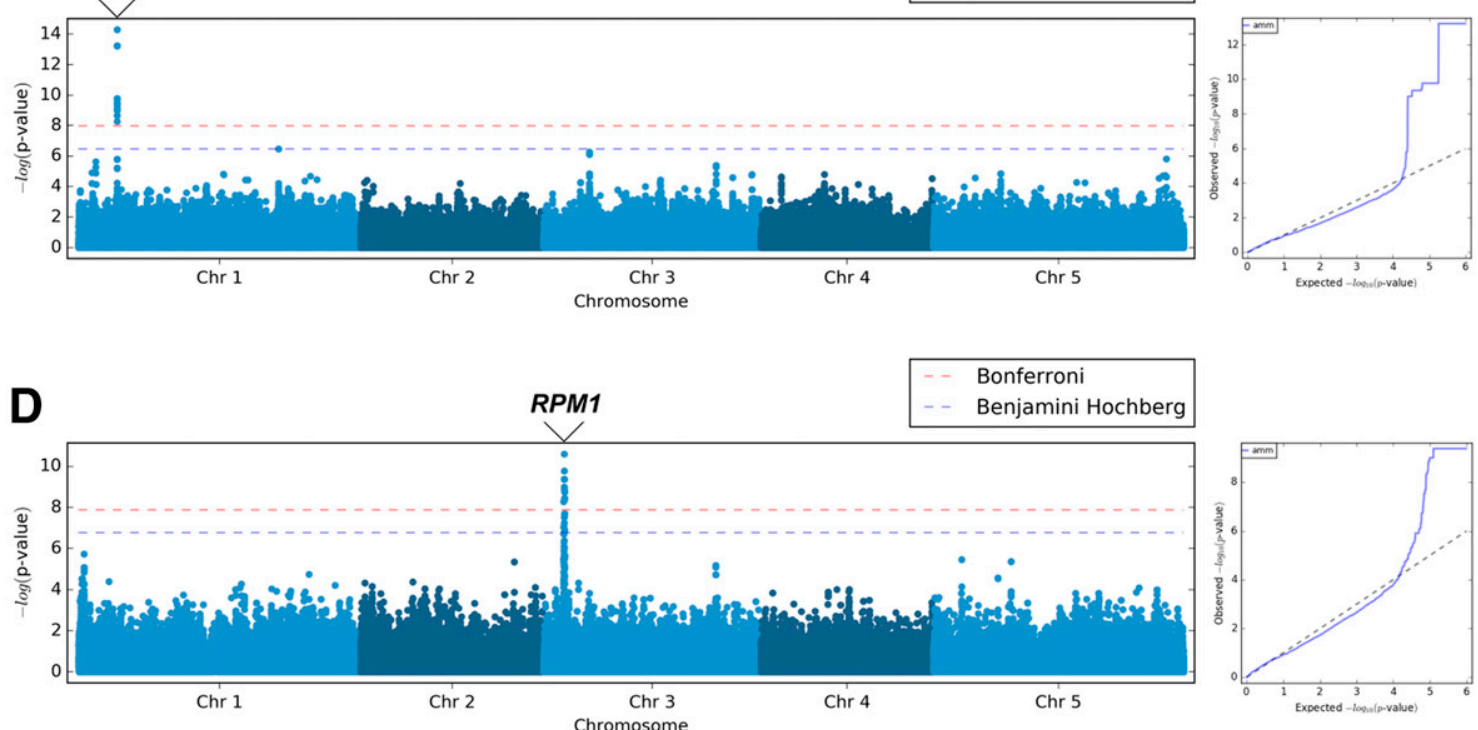

Fig. 4. Genome wide association study (GWAS) using the high-throughput seedling pathology assay successfully identifies nucleotide-binding domain leucinerich repeat receptors associated with effector-triggered immunities (ETIs) in response to HopAR1 and AvrRpm1. A, Images of the 110 Arabidopsis ecotypes screened for ETI induction against the Pseudomonas syringae type III secreted effector HopAR1. The Effects green-pass filter shows healthy green tissue in its natural color and diseased tissue in gray. B, List of all ecotypes screened for the HopAR1 GWAS according to their CS number. The background color represents whether an ecotype was considered resistant (green) or susceptible (yellow) based on Plant Immunity and Disease Image-Based Quantification (PIDIQ) quantification. The full list of ETI calls for each ecotype are presented in Supplementary Table S1. C, Manhattan and QQ plots of the HopAR1 GWAS The significant peak in chromosome 1 is located at the RPS5 locus. D, Manhattan and QQ plots of the AvrRpm1 GWAS. The significant peak in chromosome 3 is located at the RPM1 locus. The dashed lines represent the Bonferroni (red) and Benjamini Hochberg (blue) corrected significance cut-offs. 
T3SEs using a similar number of ecotypes (70 or 110 vs. 90 ecotypes) (Fig. 4C) (Atwell et al. 2010). Notably, our results were obtained by quantifying disease symptoms rather than assessing HRs (Atwell et al. 2010). The setup time, growth space, experimental length, and labor was significantly reduced with our approach. The 48-well microplate-based seedling assay required less than the space of a single plant growth tray to generate sufficient data for GWAS on the ETI elicitors tested. Furthermore, each plate required less than 3 min to set up and the overall experimental length was 3 weeks from set up to GWAS analysis. In the space required to amass $60 \mathrm{~T} 3 \mathrm{SE}$-ecotype interactions (one plant growth tray, 6 rows by 10 columns) using standard techniques, our method may assess 576 T3SE-ecotype interactions (12 plates by 48 genotypes) in less than half the time (3 weeks for the microplate assay versus at least 6 weeks for standard techniques).

Although all of our work was performed in Arabidopsis using $P$. syringae heterologously expressing T3SEs, the method may be applicable to the study of effectors from other pathogens using $P$. syringae-based translocation systems such as effector detector vectors (Sohn et al. 2007) or any pathogen amenable to spray infection, and the screen should be applicable to any plant host that can germinate and grow in very small soil volumes and with limited space. To accommodate plant species that require more growth space, larger-welled microplates may be appropriate or, alternatively, pots or multiwelled growing trays. The cropping macro associated with PIDIQ can be easily modified to accommodate images from alternative growth conditions. Furthermore, the PIDIQ parameters may be adjusted to accommodate the various symptomologies obtained when using different pathogen backgrounds or host species that do not display chlorosis as the primary visual disease symptom. We believe that this seedling pathology assay will greatly accelerate the throughput of large-scale ETI screens.

\section{MATERIALS AND METHODS}

\section{Seed dispensing.}

We created a seed dispenser that permits rapid and reproducible delivery of seed in a 48 -well format (Supplementary Fig. S2). We removed the bottom of two 48-well microplates using a heated 7-mm core puncher, then stacked and taped the two plates together for form a dispenser frame. We then removed the caps and perforated the bottoms of $0.5-\mathrm{ml}$ microcentrifuge tubes using an awl just big enough for Arabidopsis seed to pass through to create the dispenser tubes. These perforated microcentrifuge dispenser tubes were inserted into each well of the stacked microplate frame to complete the seed dispenser. Arabidopsis seed could then be added to the tubes for easy dispensing in a 48-well format (see below).

\section{Plate setup and growth conditions.}

We created bottom-watering, 48-well growth microplates by perforating the bottom of microplates with a heated awl or power drill. We used Sunshine number 3 soil mix in which large soil particles were removed by sifting the soil using a sieve with 3.35-mm apertures (Fig. 1B). These 48-well microplates were filled and leveled with sieved soil. Plates were then centrifuged at $670 \times g$ for $30 \mathrm{~s}$ using a Thermo Jouan CR3i multifunction centrifuge with the T20 rotor. We then watered the soil by placing the bottom-watering plates in a container with shallow water until all wells were saturated. Seeds were gas sterilized for $3.5 \mathrm{~h}$ using $35 \mathrm{ml}$ of $2 \times$ bleach and $2 \mathrm{ml}$ of $\mathrm{HCl}$, then dispensed into wells using the seed dispenser described above. We found that simply aligning the dispenser precisely over the growth microplates and tapping three times dispensed 1 to 25 seeds/well. Seeded plates were stratified at $4^{\circ} \mathrm{C}$ with lids on for
2 days, then placed into a growth chamber with the following conditions: 12 -h photoperiod, $150 \mu \mathrm{E}$ of light, $22^{\circ} \mathrm{C}$, and 50 to $70 \%$ humidity. Plates were placed in a standard flat and domed to maintain humidity. Eight plates fit in a standard flat covered with a dome; however, 12 plates can be covered with a dome if they are placed directly on the growth chamber's shelf. Plate lids were removed following 1 day of growth in the chamber, while the domes were retained during the entire growth period and periodically sprayed with water to maintain humidity levels.

\section{Pathogen spray assays and plant genotypes.}

Pathology assays were performed on 7-day-old seedlings or when the seedling canopy reached the top of the well. P. syringae pv. tomato DC3000 harboring T3SEs of interest under their native promoters in pBBR1-MCS2 (Kovach et al. $1995)$ or an EV control were grown on King's B agar media supplemented with rifampicin (strain selection) at $50 \mu \mathrm{g} / \mathrm{ml}$ and kanamycin (vector selection) at $50 \mu \mathrm{g} / \mathrm{ml}$ for $24 \mathrm{~h}$ at $30^{\circ} \mathrm{C}$. The freshly grown bacteria were resuspended in $10 \mathrm{mM} \mathrm{MgSO}_{4}$, diluted to an optical density at $600 \mathrm{~nm}=1$, and supplemented with $0.04 \%$ Silwet L-77. Each plate was sprayed with $10 \mathrm{ml}$ of prepared bacterial inoculum using Preval sprayers and immediately returned to domed flats. The underside of the dome was sprayed with water to maintain high humidity levels. Each plate was resprayed 2 days following the first inoculation using the same protocol. Plates were kept at room temperature under constant light, and water was periodically sprayed inside the domes to maintain high humidity levels. Plates were photographed periodically for a 10-day period with a Nikon D5200 DSLR camera fitted with a Tamron SP AF $90 \mathrm{~mm}$ f/2.8 Macro 1:1 Di Lens. To obtain images that emphasized healthy plant tissue, the EFFECTS/Selective Color option was used with green selected. This green-pass filter shows healthy tissue in its original color and diseased or chlorotic tissues as gray. Note that this filter is used only for presentation purposes to emphasize green or healthy tissue. All quantitative analyses using PIDIQ (Laflamme et al. 2016) were performed on unfiltered images (Supplementary Figs. S3, S4, S5, and S7).

The following plant knockout lines were used in this study: zarl-1 (At3g50950; SALK_013297), zed1-2 (At3g57750; EMS line), and $z r k 2-1$ (At3g55720; SAIL_916_G06) (Lewis et al. 2010, 2013). A complete list of Arabidopsis ecotypes used in this study is presented in Supplementary Table S1.

\section{Disease quantification.}

Plate images were initially cropped to only encompass the seedling-containing wells. Individual wells from each plate were subsequently cropped using our ImageJ-based cropping macro, which is is available on GitHub. Subsequently, disease symptom quantification for each cropped well was performed using PIDIQ (Laflamme et al. 2016).

\section{GWAS.}

The GWAS was performed using the above-described pathology assay with PtoDC3000::HopAR1 or PtoDC3000:: AvrRpm1 under their respective native promoters in pBBR1MCS2 on 110 Arabidopsis ecotypes or 70 Arabidopsis ecotypes, respectively (ecotypes listed in Supplementary Table S1). Images of plates at 7 to 10 dpi were used to classify ecotypes as susceptible or resistant. Wells were cropped and symptoms were quantified using the above-described methodology. Based on the obtained distributions of disease symptoms (Supplementary Figs. S6 and S8), a cut-off of 0.35 (percent yellow [arcsine transformed]) was selected to delineate susceptible $(>0.35)$ and resistant $(<0.35)$ ecotypes for HopAR1, while a cut-off of 0.60 was used for AvrRpm1. These cut-offs were chosen to separate the obtained bimodal distributions, 
specific to each T3SE tested, into susceptible and resistant subsets (Supplementary Figs. S6 and S8). Ecotype classifications based on these cut-offs (Supplementary Table S1) were used for GWAS analysis using the online Arabidopsis GWAPortal (Segura et al. 2012; Seren 2018). An accelerated mixedmodel GWAS was performed using the single-nucleotide polymorphism data from the 1,001 genomes project (Segura et al. 2012; Seren 2018; Weigel and Mott 2009).

All primary data are available from the authors upon request.

\section{ACKNOWLEDGMENTS}

We thank members of the Guttman and Desveaux labs for valuable input on the project, particularly B. Laflamme, D. Seto, M. Khan, R. Almeida, J. Diaz-Caballero, M. M. Dillon, and M. Middleton for experimental design input and script creation; and A. Severino and P. W. Wang for ecotype bulking and seed collection.

\section{AUTHOR-RECOMMENDED INTERNET RESOURCES}

ImageJ cropping macro: https://github.com/DSGlab/Crop-macro GWA-Portal: https://gwas.gmi.oeaw.ac.at/

\section{LITERATURE CITED}

Abramovitch, R. B., Anderson, J. C., and Martin, G. B. 2006. Bacterial elicitation and evasion of plant innate immunity. Nat. Rev. Mol. Cell Biol. 7:601-611.

Atwell, S., Huang, Y. S., Vilhjálmsson, B. J., Willems, G., Horton, M., Li, Y., Meng, D., Platt, A., Tarone, A. M., Hu, T. T., Jiang, R., Muliyati, N. W., Zhang, X., Amer, M. A., Baxter, I., Brachi, B., Chory, J., Dean, C., Debieu, M., de Meaux, J., Ecker, J. R., Faure, N., Kniskern, J. M., Jones, J. D., Michael, T., Nemri, A., Roux, F., Salt, D. E., Tang, C., Todesco, M., Traw, M. B., Weigel, D., Marjoram, P., Borevitz, J. O., Bergelson, J., and Nordborg, M. 2010. Genome-wide association study of 107 phenotypes in Arabidopsis thaliana inbred lines. Nature 465: 627-631.

Baltrus, D. A., McCann, H. C., and Guttman, D. S. 2017. Evolution, genomics and epidemiology of Pseudomonas syringae: Challenges in bacterial molecular plant pathology. Mol. Plant Pathol. 18: $152-168$.

Berge, O., Monteil, C. L., Bartoli, C., Chandeysson, C., Guilbaud, C., Sands, D. C., and Morris, C. E. 2014. A user's guide to a data base of the diversity of Pseudomonas syringae and its application to classifying strains in this phylogenetic complex. PLoS One 9:e105547.

Büttner, D. 2016. Behind the lines-actions of bacterial type III effector proteins in plant cells. FEMS Microbiol. Rev. 40:894-937.

Couto, D., and Zipfel, C. 2016. Regulation of pattern recognition receptor signalling in plants. Nat. Rev. Immunol. 16:537-552.

Cui, H., Tsuda, K., and Parker, J. E. 2015. Effector-triggered immunity: From pathogen perception to robust defense. Annu. Rev. Plant Biol. 66: 487-511.

Dillon, M. M., Almeida, R. N. D., Laflamme, B., Martel, A., Weir, B. S., Desveaux, D., and Guttman, D. S. 2019a. Molecular evolution of Pseudomonas syringae type III secreted effector proteins. Front. Plant Sci. 10:418.

Dillon, M. M., Thakur, S., Almeida, R. N. D., Wang, P. W., Weir, B. S., and Guttman, D. S. 2019b. Recombination of ecologically and evolutionarily significant loci maintains genetic cohesion in the Pseudomonas syringae species complex. Genome Biol. 20:3.

Fan, J., Crooks, C., and Lamb, C. 2008. High-throughput quantitative luminescence assay of the growth in planta of Pseudomonas syringae chromosomally tagged with Photorhabdus luminescens luxCDABE. Plant J. 53:393-399.

Gassmann, W., Hinsch, M. E., and Staskawicz, B. J. 1999. The Arabidopsis RPS4 bacterial-resistance gene is a member of the TIR-NBS-LRR family of disease-resistance genes. Plant J. 20:265-277.

Grant, M. R., Godiard, L., Straube, E., Ashfield, T., Lewald, J., Sattler, A., Innes, R. W., and Dangl, J. L. 1995. Structure of the Arabidopsis RPM1 gene enabling dual specificity disease resistance. Science 269: 843-846.

Huard-Chauveau, C., Perchepied, L., Debieu, M., Rivas, S., Kroj, T., Kars, I., Bergelson, J., Roux, F., and Roby, D. 2013. An atypical kinase under balancing selection confers broad-spectrum disease resistance in Arabidopsis. PLoS Genet. 9:e1003766.
Jayaraman, J., Choi, S., Prokchorchik, M., Choi, D. S., Spiandore, A., Rikkerink, E. H., Templeton, M. D., Segonzac, C., and Sohn, K. H. 2017 A bacterial acetyltransferase triggers immunity in Arabidopsis thaliana independent of hypersensitive response. Sci. Rep. 7:3557.

Jones, J. D., and Dangl, J. L. 2006. The plant immune system. Nature 444 323-329.

Khan, M., Seto, D., Subramaniam, R., and Desveaux, D. 2018. Oh, the places they'll go! A survey of phytopathogen effectors and their host targets. Plant J. 93:651-663.

Kim, S. H., Kwon, S. I., Saha, D., Anyanwu, N. C., and Gassmann, W. 2009. Resistance to the Pseudomonas syringae effector HopA1 is governed by the TIR-NBS-LRR protein RPS6 and is enhanced by mutations in SRFR1. Plant Physiol. 150:1723-1732.

Kovach, M. E., Elzer, P. H., Hill, D. S., Robertson, G. T., Farris, M. A., Roop, R. M., II, and Peterson, K. M. 1995. Four new derivatives of the broad-host-range cloning vector pBBR1MCS, carrying different antibiotic-resistance cassettes. Gene 166:175-176.

Kunkel, B. N., Bent, A. F., Dahlbeck, D., Innes, R. W., and Staskawicz, B. J. 1993. RPS2, an Arabidopsis disease resistance locus specifying recognition of Pseudomonas syringae strains expressing the avirulence gene avrRpt2. Plant Cell 5:865-875.

Laflamme, B., Middleton, M., Lo, T., Desveaux, D., and Guttman, D. S. 2016. Image-based quantification of plant immunity and disease. Mol. Plant-Microbe Interact. 29:919-924.

Lewis, J. D., Abada, W., Ma, W., Guttman, D. S., and Desveaux, D. 2008 The HopZ family of Pseudomonas syringae type III effectors require myristoylation for virulence and avirulence functions in Arabidopsis thaliana. J. Bacteriol. 190:2880-2891.

Lewis, J. D., Lee, A. H., Hassan, J. A., Wan, J., Hurley, B., Jhingree, J. R., Wang, P. W., Lo, T., Youn, J. Y., Guttman, D. S., and Desveaux, D. 2013. The Arabidopsis ZED1 pseudokinase is required for ZAR1-mediated immunity induced by the Pseudomonas syringae type III effector HopZ1a. Proc. Natl. Acad. Sci. U.S.A. 110:18722-18727.

Lewis, J. D., Wu, R., Guttman, D. S., and Desveaux, D. 2010. Allelespecific virulence attenuation of the Pseudomonas syringae HopZ1a type III effector via the Arabidopsis ZAR1 resistance protein. PLoS Genet. 6:e1000894.

Ma, W., Dong, F., Stavrinides, J., and Guttman, D. S. 2006. Type III effector diversification via both pathoadaptation and horizontal transfer in response to a coevolutionary arms race. PLoS Genet. 2:e209.

Mansfield, J., Genin, S., Magori, S., Citovsky, V., Sriariyanum, M., Ronald, P., Dow, M., Verdier, V., Beer, S. V., Machado, M. A., Toth, I., Salmond, G., and Foster, G. D. 2012. Top 10 plant pathogenic bacteria in molecular plant pathology. Mol. Plant Pathol. 13:614-629.

Mittal, S., and Davis, K. R. 1995. Role of the phytotoxin coronatine in the infection of Arabidopsis thaliana by Pseudomonas syringae pv. tomato. Mol. Plant-Microbe Interact. 8:165-171.

Morris, C. E., Monteil, C. L., and Berge, O. 2013. The life history of Pseudomonas syringae: Linking agriculture to earth system processes. Annu. Rev. Phytopathol. 51:85-104.

Provart, N. J., Alonso, J., Assmann, S. M., Bergmann, D., Brady, S. M., Brkljacic, J., Browse, J., Chapple, C., Colot, V., Cutler, S., Dangl, J., Ehrhardt, D., Friesner, J. D., Frommer, W. B., Grotewold, E., Meyerowitz, E., Nemhauser, J., Nordborg, M., Pikaard, C., Shanklin, J., Somerville, C., Stitt, M., Torii, K. U., Waese, J., Wagner, D., and McCourt, P. 2016. 50 Years of Arabidopsis research: Highlights and future directions. New Phytol. 209:921-944.

Segura, V., Vilhjálmsson, B. J., Platt, A., Korte, A., Seren, Ü., Long, Q., and Nordborg, M. 2012. An efficient multi-locus mixed-model approach for genome-wide association studies in structured populations. Nat. Genet. 44:825-830.

Seren, Ü. 2018. GWA-Portal: Genome-wide association studies made easy. Methods Mol. Biol. 1761:303-319.

Seren, Ü., Vilhjálmsson, B. J., Horton, M. W., Meng, D., Forai, P., Huang, Y. S., Long, Q., Segura, V., and Nordborg, M. 2012. GWAPP: A web application for genome-wide association mapping in Arabidopsis. Plant Cell 24:4793-4805

Seto, D., Koulena, N., Lo, T., Menna, A., Guttman, D. S., and Desveaux, D. 2017. Expanded type III effector recognition by the ZAR1 NLR protein using ZED1-related kinases. Nat. Plants 3:17027.

Smakowska-Luzan, E., Mott, G. A., Parys, K., Stegmann, M., Howton, T. C., Layeghifard, M., Neuhold, J., Lehner, A., Kong, J., Grünwald, K., Weinberger, N., Satbhai, S. B., Mayer, D., Busch, W., Madalinski, M., Stolt-Bergner, P., Provart, N. J., Mukhtar, M. S., Zipfel, C., Desveaux, D., Guttman, D. S., and Belkhadir, Y. 2018. An extracellular network of Arabidopsis leucine-rich repeat receptor kinases. Nature 553:342-346 
Sohn, K. H., Lei, R., Nemri, A., and Jones, J. D. 2007. The downy mildew effector proteins ATR1 and ATR13 promote disease susceptibility in Arabidopsis thaliana. Plant Cell 19:4077-4090.

Warren, R. F., Henk, A., Mowery, P., Holub, E., and Innes, R. W. 1998. A mutation within the leucine-rich repeat domain of the Arabidopsis disease resistance gene RPS5 partially suppresses multiple bacterial and downy mildew resistance genes. Plant Cell 10:1439-1452.

Weigel, D., and Mott, R. 2009. The 1001 genomes project for Arabidopsis thaliana. Genome Biol. 10:107.
Wu, C. H., Abd-El-Haliem, A., Bozkurt, T. O., Belhaj, K., Terauchi, R., Vossen, J. H., and Kamoun, S. 2017. NLR network mediates immunity to diverse plant pathogens. Proc. Natl. Acad. Sci. U.S.A. 114:8113-8118.

Xin, X. F., Kvitko, B., and He, S. Y. 2018. Pseudomonas syringae: What it takes to be a pathogen. Nat. Rev. Microbiol. 16:316-328.

Yu, G. L., Katagiri, F., and Ausubel, F. M. 1993. Arabidopsis mutations at the RPS2 locus result in loss of resistance to Pseudomonas syringae strains expressing the avirulence gene avrRpt2. Mol. Plant-Microbe Interact. 6:434-443. 